\title{
LA-UR-16-25919
}

Approved for public release; distribution is unlimited.

Title:

Author(s):

Intended for:

Issued:
Analysis of Trinity Power Metrics for Automated Monitoring

Michalenko, Ashley Christine

Share with my university

2016-08-02 
Disclaimer:

Los Alamos National Laboratory, an affirmative action/equal opportunity employer, is operated by the Los Alamos National Security, LLC for the National Nuclear Security Administration of the U.S. Department of Energy under contract DE-AC52-06NA25396. By approving this article, the publisher recognizes that the U.S. Government retains nonexclusive, royalty-free license to publish or reproduce the published form of this contribution, or to allow others to do so, for U.S. Government purposes. Los Alamos National Laboratory requests that the publisher identify this article as work performed under the auspices of the U.S. Department of Energy. Los Alamos National Laboratory strongly supports academic freedom and a researcher's right to publish; as an institution, however, the Laboratory does not endorse the viewpoint of a publication or guarantee its technical correctness. 


\section{Analysis of Trinity Power Metrics for Automated Monitoring}

Ashley Michalenko

July 28,2016 


\section{Outline}

- Current monitoring efforts

- Motivation for analysis

- Tools used

- Methodology

- Summer work

- Future work 


\section{Current Monitoring Infrastructure}

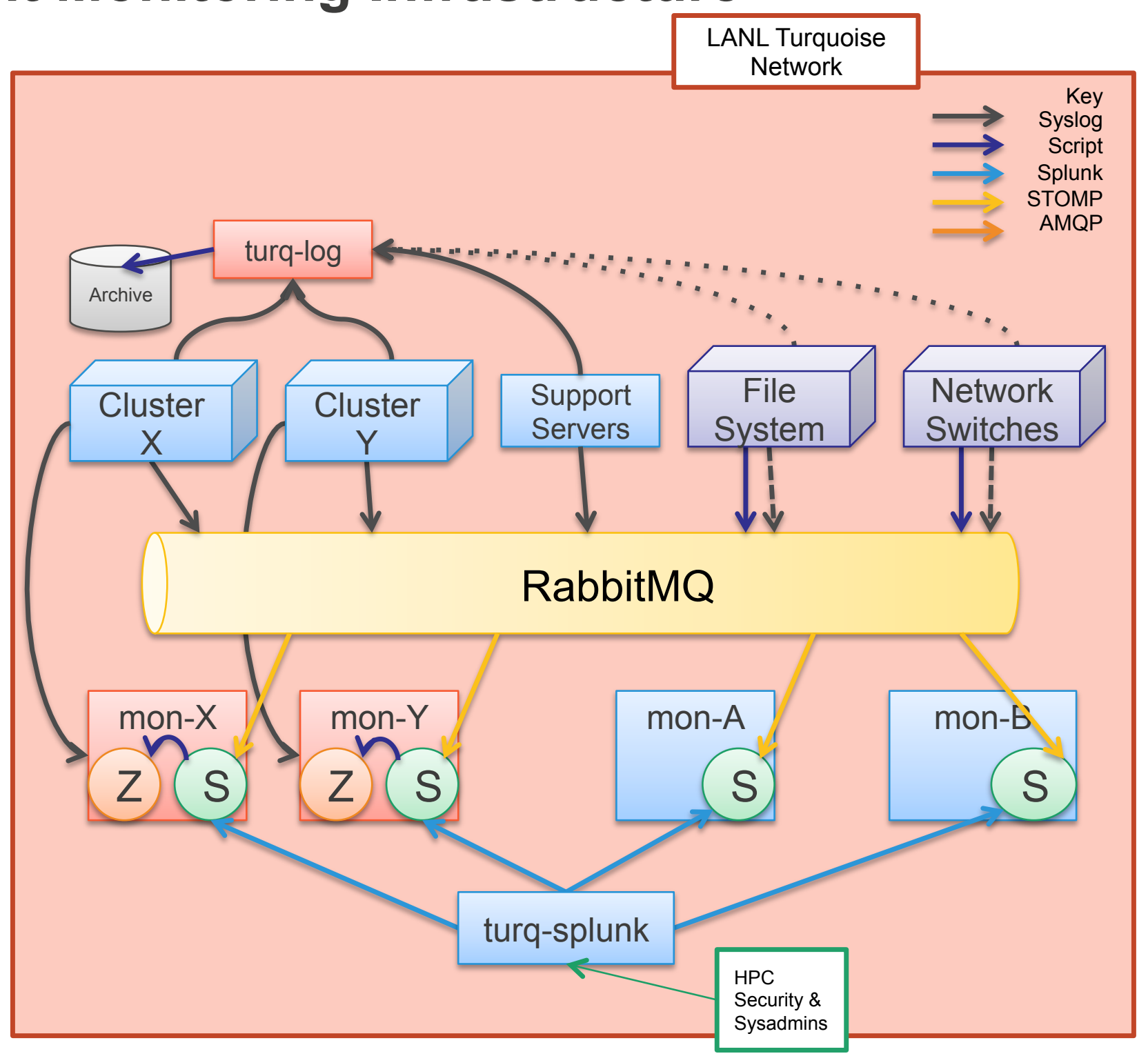




\section{Motivation for enhanced monitoring}

- Current monitoring efforts will not be sufficient for future machines

- These applications will not be able to handle the amount of data that Trinity and future systems will produce

- Admin's do not have a full system view of statistics 


\section{Enhanced monitoring: LDMS}

- Run LDMS on every compute node

- Continuous monitoring

- LDMS pulls detailed metrics about the systems state 


\section{LDMS}

- Application out of SNL

- Collects metrics for memory, CPU, power, Cray Aries Network, etc.

- Set to collect once every 10 seconds

- Collected data on Trinity during open science period

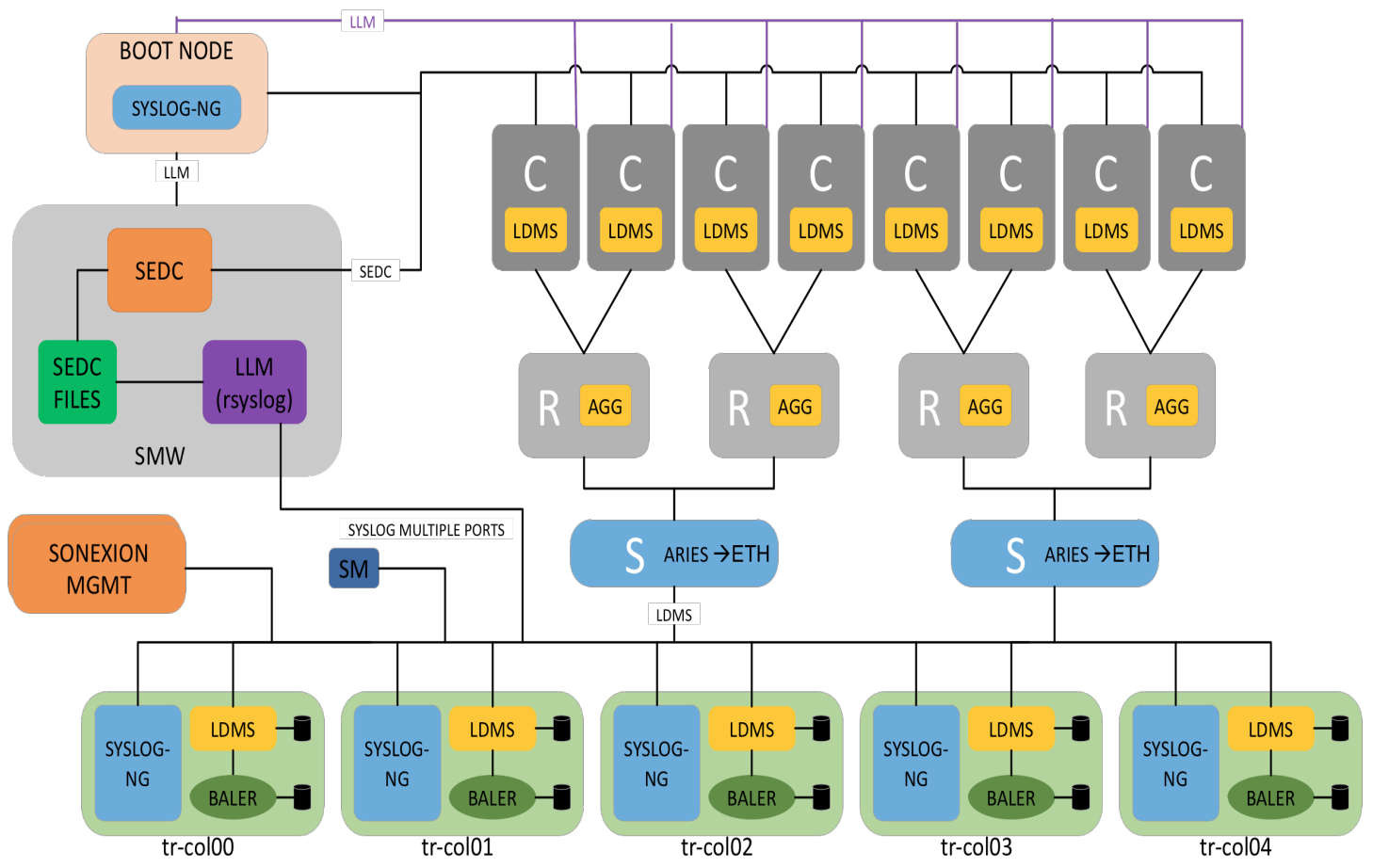




\section{Why Machine Learning?}

- Can handle large amounts of data

- Estimated to produce $4 \mathrm{~TB} /$ day of data

- Compared to $40 \mathrm{~GB} /$ day of syslog

- Automated anomaly detection

- Saves time

- There is no need to save all data, just data for events of interest 


\section{What does LDMS output look like?}

$0,365253551,365253591,365253631,365253280,365253314,365253353,365253393,365253433,365253472,365253512,397,398,422,388,401,420,377,385,394,384$ $0,365672847,365672885,365672885,365672962,365673000,365672655,365672693,365672731,365672770,365672808,368,388,388,397,369,393,396,367,385,379$ $0,352091595,352091216,352091291$, 352091329, 352091368, 352091406, 352091406, 352091482, 352091520, 352091558, 387, 363, 391, $372,363,388,388,368,381,395$ $0,348161260,348160935,348160971,348161008,348161043,348161079,348161116,348161152,348161188,348161224,358,358,357,356,370,363,357,358,358,358$ $0,92949308,92949316,92949323,92949330,92949336,92949342,92949272,92949282,92949292,92949300,58,68,61,75,69,65,66,63,68,61$ $0,85345050,85345060,85345068,85345076,85345000,85345008,85345017,85345025,85345033,85345042,90,89,94,81,64,70,78,104,103,91$ $0,99310993,99311009,99311027,99311041,99311057,99310916,99310932,99310949,99310962,99310978,157,159,165,146,171,165,152,153,166,163$ $0,89773490,89773499,89773506,89773513,89773521,89773529,89773538,89773545,89773553,89773483,73,75,89,81,75,66,84,85,95,83$

$0,329840144,329840178,329840213,329840247,329839938,329839972,329840006,329840040,329840074,329840109,341,341,340,343,341,341,340,358,340,341$ $0,347877154,347876830,347876865,347876901,347876936,347876973,347877012,347877045,347877081,347877118,362,359,360,360,368,377,361,362,360,361$ $0,347874495,347874170,347874206,347874242,347874278,347874314,347874350,347874387,347874423,347874459,361,361,361,359,355,365,373,358,359,360$ $0,358905004,358905040,358905076,358905113,358905149,358905185,358904859,358904895,358904931,358904967,361,362,363,362,361,362,363,362,363,373$ $0,352587462,352587498,352587534,352587570,352587607,352587643,352587317,352587353,352587389,352587425,376,363,361,363,362,361,361,363,361,359$ $0,347277306,347277342,347277377,347277413,347277448,347277129,347277165,347277200,347277235,347277271,354,353,353,354,354,353,355,354,354,366$ $0,363576066,363576094,363576131,363575804,363575842$, 363575879, 363575916, 363575954, 363575991, 363576029, 373, 372, 373, 371, 371, 369, $380,387,372,373$ $0,358824832,358824868,358824905,358824942,358824979,358825015,358825052,358824723,358824759,358824796,364,378,381,364,365,365,363,364,365,366$ $0,358163704,358163743,358163777,358163814,358163851,358163888,358163928,358163594,358163630,358163667,366,375,371,368,366,368,366,368,366,367$ $0,353252898,353252935,353252975,353252645,353252681,353252718,353252754,353252792,353252826,353252862,362,362,361,361,361,357,374,364,360,362$ $0,356026690,356026728,356026762,356026798,356026834,356026871,356026910,356026943,356026618,356026654,359,365,367,362,362,361,361,360,360,360$ $0,347532920,347532588,347532624,347532660,347532697,347532735,347532770,347532806,347532843,347532880,365,365,365,363,372,381,362,364,363,365$ $0,345997337,345997372,345997408,345997444,345997121,345997157,345997193,345997229,345997264,345997300,359,357,359,358,356,356,356,372,359,358$ $0,56701460,56701460,56701460,56701460,56701460,56701460,56701482,56701482,56701482,56701460,44,44,44,44,44,44,44,44,44,44$ $0,55194081,55194081,55194081,55194102,55194102,55194102,55194102,55194059,55194059,55194081,44,44,44,44,44,44,44,44,44,44$ $0,354353429,354353100,354353137,354353178,354353209,354353246,354353282,354353319,354353356,354353392,361,360,363,362,364,379,363,362,364,363$ $0,353747701,353747738,353747775,353747811,353747481,353747518,353747554,353747591,353747627,353747664,367,368,367,367,366,365,365,368,376,366$ $0,342111659,342111694,342111730,342111765,342111447,342111482,342111517,342111553,342111588,342111623,349,349,349,350,350,350,347,360,353,349$ $0,340514105,340514141,340514176,340514211,340514247,340514283,340514319,340514354,340514035,340514070,349,367,359,349,350,350,349,351,348,350$ $0,341686981,341687017,341687054,341687089,341687125,341687162,341687198,341687234,341686910,341686945,357,360,367,358,360,361,359,356,357,356$ $0,338511940,338511975,338512011,338512045,338512081,338511765,338511800,338511835,338511870,338511905,354,346,347,346,346,347,347,347,343,361$ $0,349021720,349021756,349021792,349021828,349021542,349021542,349021583,349021613,349021648,349021684,355,355,357,355,353,355,354,363,357,357$ $0,344437570,344437606,344437642,344437678,344437353,344437389,344437425,344437461,344437497,344437534,356,356,357,357,358,358,357,353,375,376$ 


\section{LDMS Headers}

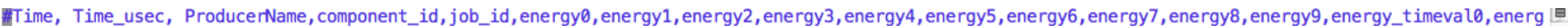
y_timeval1, energy_timeval2, energy_timeval3, energy_timeval4, energy_timeval5, energy_timeval6, energy_timeval7, energy_timeval8, energy_timeval9, energy_timeva 110, energy_timeval11, energy_timeval12, energy_timeval13, energy_timeval14, energy_timeval15, energy_timeval16, energy_timeval17, energy_timeval18, energy_timev al19, power0, power1, power2, power3, power4, power5, power6, power7, power8, power9, power_timeval0, power_timeval1, power_timeval2, power_timeval3, power_timeval4, pc wer_timeval5, power_timeval6, power_timeval7, power_timeval8, power_timeval9, power_timeval10, power_timeval11, power_timeval12, power_timeval13, power_timeval14 , power_timeval15, power_timeval16, power_timeval17, power_timeval18, power_timevalis $\sim$ $\sim$

\#Time, Time_usec, ProducerName, component_id, job_id, aries_rtr_id, AR_NIC_NETMON_ORB_EVENT_CNTR_REQ_PKTS, AR_NIC_NETMON_ORB_EVENT_CNTR_REQ_FLITS, AR_NIC_NETM I I ON_ORB_EVENT_CNTR_REQ_STALLED, AR_NIC_RSPMON_PARB_EVENT_CNTR_PI_PKTS, AR_NIC_RSPMON_PARB_EVENT_CNTR_PI_FLITS, AR_NIC_RSPMON_PARB_EVENT_CNTR_PI_STALLED, AR_N IC_RSPMON_PARB_EVENT_CNTR_AMO_PKTS, AR_NIC_RSPMON_PARB_EVENT_CNTR_AMO_FLITS, AR_NIC_RSPMON_PARB_EVENT_CNTR_AMO_BLOCKED, AR_NIC_RSPMON_PARB_EVENT_CNTR_WC_PK TS, AR_NIC_RSPMON_PARB_EVENT_CNTR_WC_FLITS, AR_NIC_RSPMON_PARB_EVENT_CNTR_WC_BLOCKED, AR_NIC_RSPMON_PARB_EVENT_CNTR_BTE_RD_PKTS, AR_NIC_RSPMON_PARB_EVENT_CN TR_BTE_RD_FLITS, AR_NIC_RSPMON_PARB_EVENT_CNTR_BTE_RD_BLOCKED, AR_NIC_RSPMON_PARB_EVENT_CNTR_IOMMU_PKTS, AR_NIC_RSPMON_PARB_EVENT_CNTR_IOMMU_FLITS, AR_NIC_R SPMON_PARB_EVENT_CNTR_IOMMU_BLECKED 


\section{Data Analytics Accelerated Library - DAAL}

- Library created by Intel

- Designed and optimized for Intel processors (such as Trinity)

- Contains a set of basic machine learning algorithms

- MPI Based

- Preferred over other distributed configurations 


\section{DAAL Cont.}

\section{- Excerpt from a k-means example file}

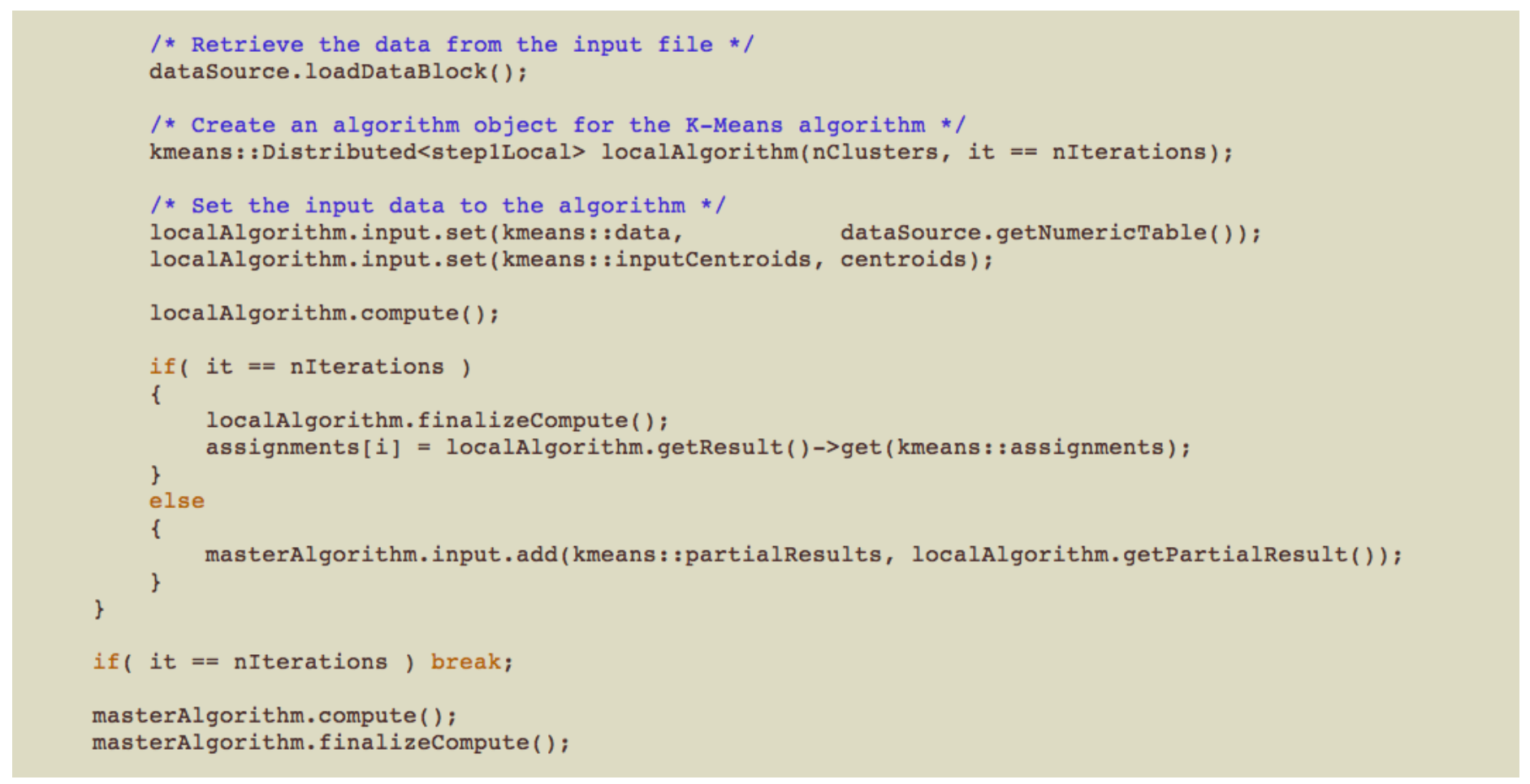




\section{Methodology}

- First step is to reduce the data set

- Remove information that is not necessary for baseline analysis

- Use two algorithms

- K-means clustering

- Cluster based on node activity

- Principal Component Analysis

- Use variance to detect anomalies and check that clustering is working correctly

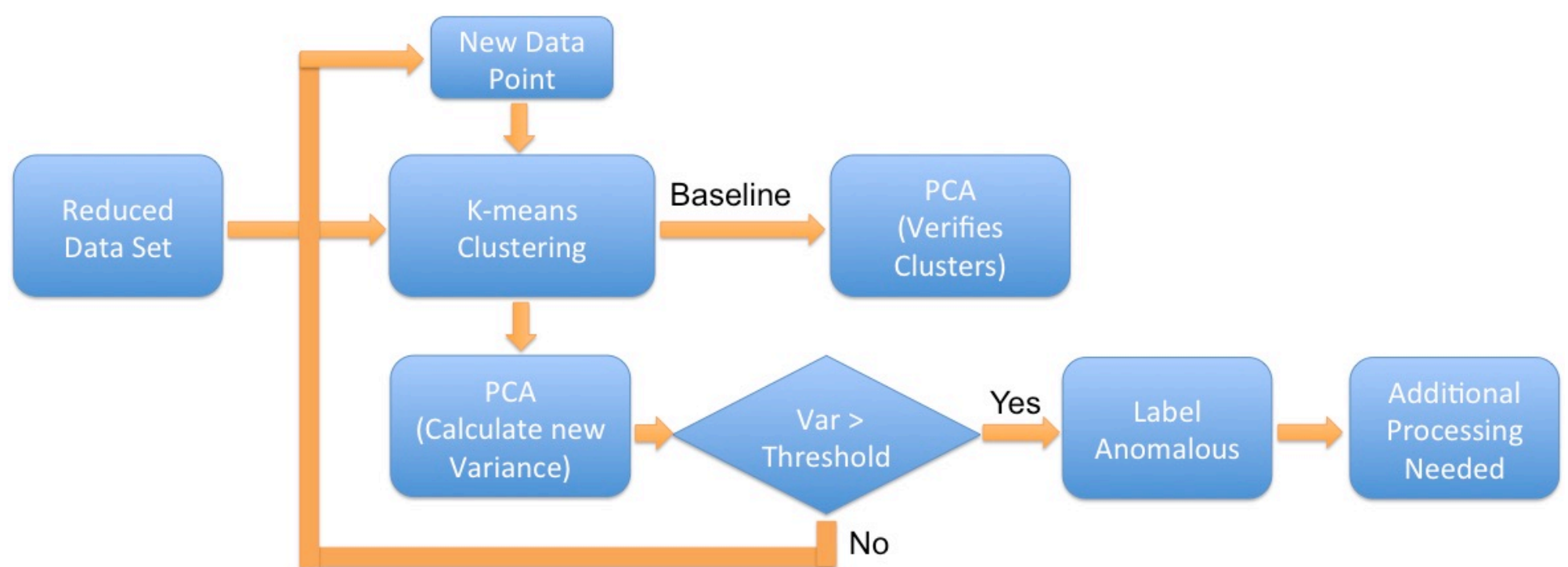




\section{Reducing Dimensions}

- Remove unnecessary features

- Timestamps and node descriptors

- When looking at Cray power sampler we reduce the dimensions of the data set from 65 to 20

- The reduced file size allows for faster analysis 


\section{K-means Clustering}

- Cluster together similar data points

- User can choose how many clusters to make

- We expect to form clusters based on node activity

- Active vs. idle node

- Type of activity/process on the node

- Power consumption should be different between these types of applications
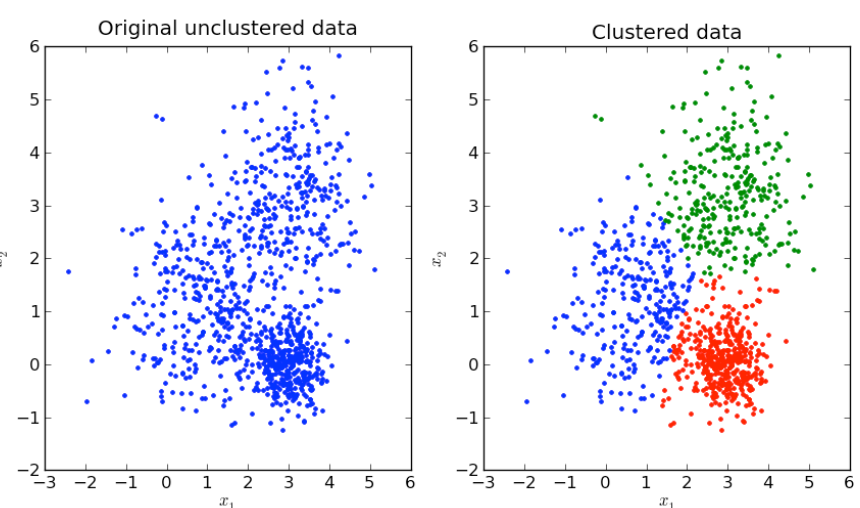


\section{K-means applied to LDMS Power Data}

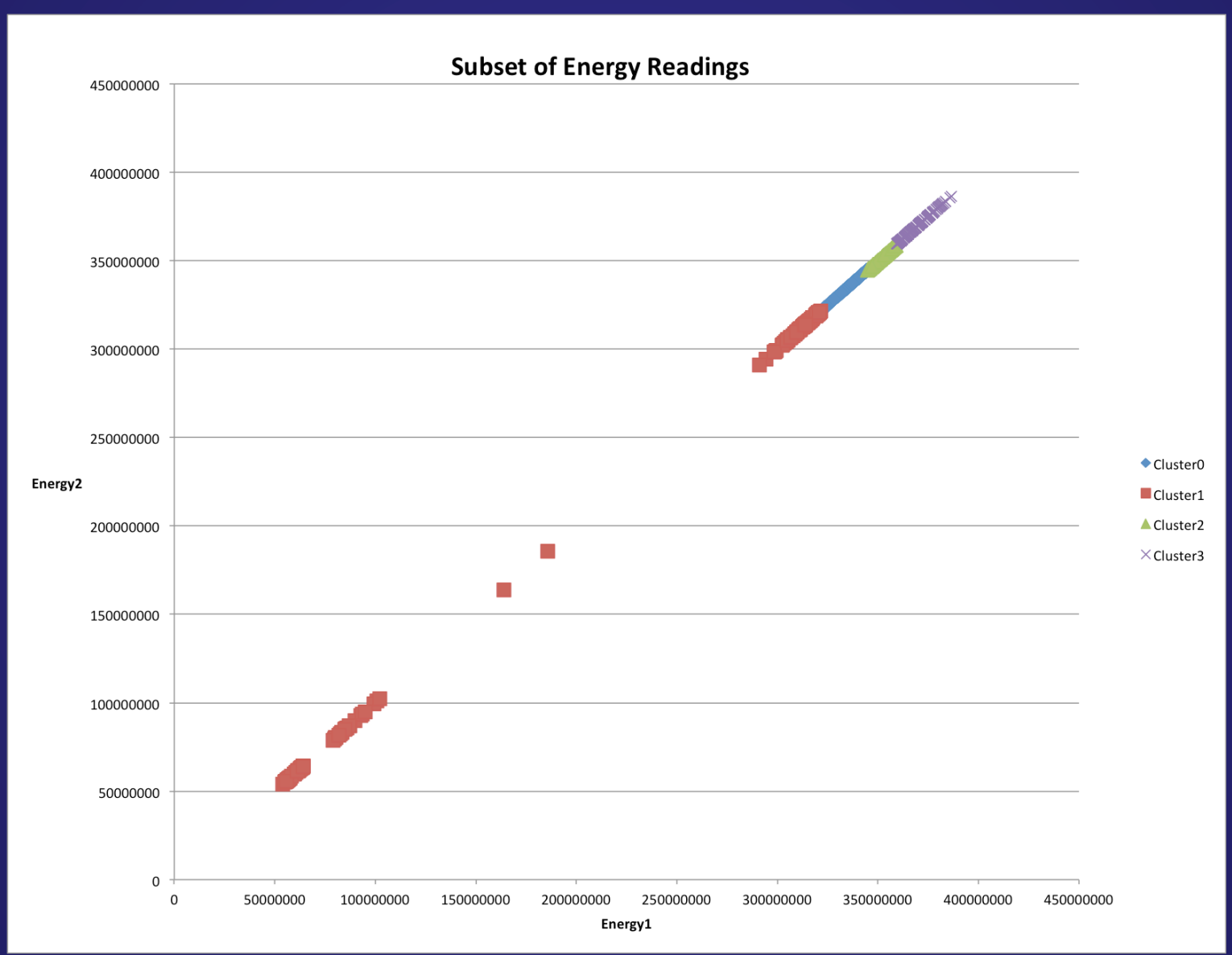




\section{Principal Component Analysis - PCA}

- Measures variance of dimensions within a data set

- Normally used to reduce dimensions

- Perform PCA on each of the clusters

- Use variance to determine if we have clustered correctly

- In future work use variance to detect anomalies

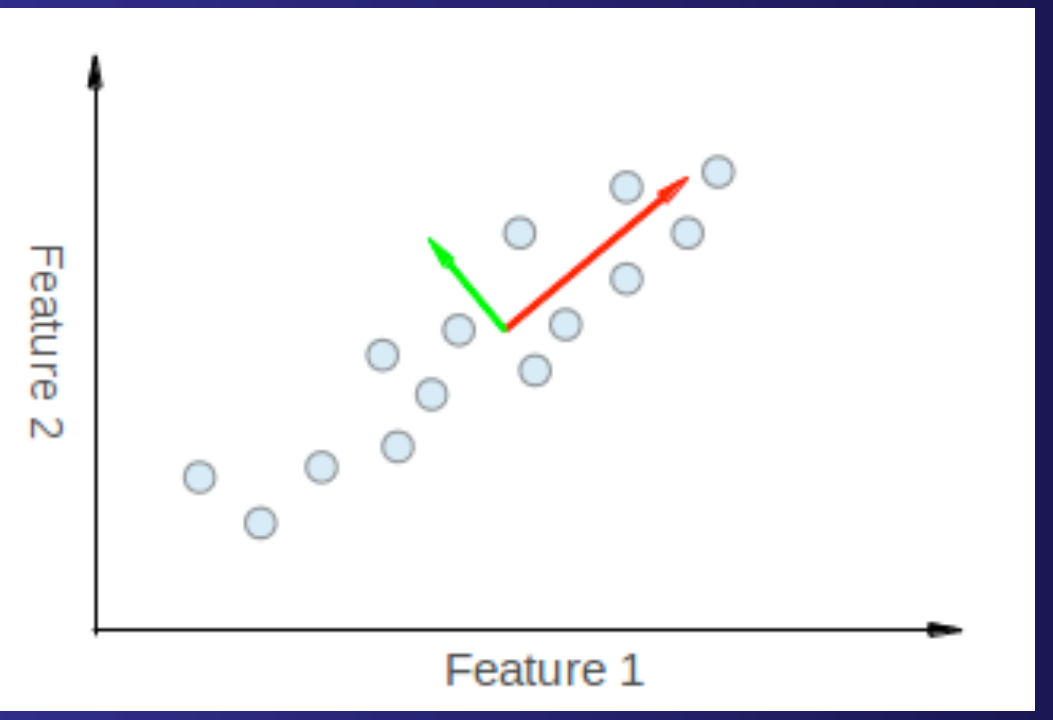




\section{Baseline}

- Goal is to use these algorithms to create a baseline for the system

- Model performance throughout the day

- Baseline can be compared with future data to determine anomalies 


\section{Goal of Summer work}

- Become familiar with DAAL, machine learning algorithms

- Work with MPI, Woodchuck

- Compute Trinity power consumption baselines 


\section{Future work}

- Continue developing baselines

- Expand analysis to other datasets

- Memory, CPU, power, Cray Aries Network, etc.

- Analyze efficiency of DAAL for our work

- Compare with other distributed set ups

- Compare with other ML algorithms 


\section{Questions? \\ Questions?}

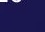

